\title{
ENRICHMENT AND PRESERVED FUNCTIONALITY OF MULTIPOTENTIAL STROMAL CELLS IN BONE MARROW CONCENTRATE PROCESSED BY VERTICAL CENTRIFUGATION
}

\author{
J.J. El-Jawhari ${ }^{1,2,3}$, D.C. Ilas ${ }^{1}$, W. Jones ${ }^{1}$, R. Cuthbert ${ }^{1}$, E. Jones ${ }^{1}$ and P.V. Giannoudis ${ }^{1,4, *}$ \\ ${ }^{1}$ University of Leeds, Leeds Institute of Rheumatic and Musculoskeletal Medicine, School of Medicine, \\ Leeds, West Yorkshire, UK \\ ${ }^{2}$ Nottingham Trent University, Department of Biosciences, School of Science and Technology, \\ Nottingham, Nottinghamshire, UK \\ ${ }^{3}$ Mansoura University, Clinical pathology department, Mansoura, Egypt \\ ${ }^{4}$ University of Leeds, Faculty of Medicine and Health, Academic Department of Trauma and Orthopaedic, \\ Leeds, West Yorkshire, UK
}

\begin{abstract}
The concentration of bone marrow (BM) aspirate (BMA) is increasingly valued for bone and cartilage repair, particularly with the rarity and donor-variability of BM-multipotential stromal cells (BM-MSCs). The present study aimed to assess BM-MSC yield following BM concentration using a fast and compact-sized vertical centrifugation system. BMA concentrate (BMAC) was separated in a $1 \mathrm{~min}$ process and collected easily after an automatic discarding of plasma and red blood cells. A significant increase in $C D 45^{\text {low }} \mathrm{CD} 271^{\text {high }}$ cells per BMAC volume (measured using flow-cytometry) was noted (4-fold, $p=0.0001$ ). Additionally, the vertical centrifugation system helped to enrich colony numbers (assessed by CFU-F assays) in BMAC comparably with conventional centrifugation systems, BioCUE ${ }^{\mathrm{TM}}$ and SmartPReP-2 ${ }^{\circledR}$ (4.3-fold, 4.6-fold and 3 -fold, respectively). Next, a functional assessment of BM-MSCs processed by vertical centrifugation was performed, and MSC viability and proliferation were not affected. Also, these BM-MSCs showed similar alkaline phosphatase and calcium levels to those of BMA-MSCs when osteogenically induced. Furthermore, glycosaminoglycans and Nile red levels in addition to the gene expression assays confirmed that there was no significant change in chondrogenic or adipogenic abilities between BMA-MSCs and BMAC-MSCs. The expression levels of selected angiogenic and immunomodulatory mediators were also similar between the two groups. Collectively, the vertical centrifugation system helped to enrich BM-MSCs effectively, while maintaining cell viability and functions. Thus, such a vertical centrifugation system for BM concentration can be valuable for various regenerative therapies.
\end{abstract}

Keywords: Multipotential stromal cells, bone marrow concentration, vertical centrifugation, regeneration, osteogenesis, chondrogenesis, angiogenesis, immunomodulation.

*Address for correspondence: Peter V. Giannoudis MD, FACS, FRCS, Professor and Chairman, Academic Unit of Trauma and Orthopaedic Surgery/Honorary Orthopaedic and Trauma Consultant, Leeds General Infirmary, School of Medicine, University of Leeds, Leeds, UK.

Telephone number: +44 1133922750 Fax number: +44 1133923290 Email: pgiannoudi@aol.com

Copyright policy: This article is distributed in accordance with Creative Commons Attribution Licence (http://creativecommons.org/licenses/by-sa/4.0/).

\begin{tabular}{|c|c|c|c|}
\hline & List of Abbreviations & $\begin{array}{l}\text { CFU-F } \\
\text { DAPI }\end{array}$ & $\begin{array}{l}\text { colony forming unit-fibroblast } \\
\text { 4' 6-diamidino-2-phenvlindole }\end{array}$ \\
\hline$A C A N$ & aggrecan & EDTA & ethylenediaminetetraacetic acid \\
\hline $\begin{array}{l}\text { ALP } \\
\text { ANGP1 }\end{array}$ & $\begin{array}{l}\text { alkaline phosphatase } \\
\text { angiopoietin } 1\end{array}$ & ELISA & $\begin{array}{l}\text { enzyme-linked immunosorbent } \\
\text { assay }\end{array}$ \\
\hline BM & bone marrow & FACS & fluorescence-activated cell sorting \\
\hline BMA & BM aspirate & FMO & fluorescence minus one \\
\hline BMAC & BMA concentrate & GAG & glycosaminoglycan \\
\hline BM-MSC & BM-multipotential stromal cell & HPRT1 & hypoxanthine \\
\hline BMP2 & bone morphogenetic protein 2 & & phosphoribosyltransferase 1 \\
\hline
\end{tabular}




$\begin{array}{ll}\text { LEPR } & \text { leptin receptor } \\ \text { MACS } & \text { magnetic-activated cell sorting } \\ \text { MFI } & \text { mean fluorescence intensity } \\ \text { MSCs } & \text { multipotential stromal cells } \\ \text { PBS } & \text { phosphate-buffered saline } \\ \text { PRP } & \text { platelet-rich plasma } \\ \text { PGE2 } & \text { prostaglandin E2 } \\ \text { PPARG } & \text { peroxisome proliferator-activated } \\ & \text { receptor gamma } \\ \text { PRP } & \text { platelet-rich plasma } \\ \text { PTGES2 } & \text { prostaglandin E synthase 2 } \\ \text { RBC } & \text { red blood cell } \\ \text { RGB } & \text { red green blue } \\ \text { ROS } & \text { reactive oxygen species } \\ \text { SOX9 } & \text { SRY-box } 9 \\ \text { SPARC } & \text { osteonectin } \\ \text { SPP1 } & \text { osteopontin } \\ \text { TGF } \beta & \text { transforming growth factor beta } \\ \text { VEGF } & \text { vascular endothelial growth factor } \\ \text { WBC } & \text { white blood cell }\end{array}$

\section{Introduction}

BMA as a standard source of MSCs has considerable potential for bone, cartilage and other musculoskeletal tissue regenerative therapies (Gaul et al., 2019; Verboket et al., 2018). BM-MSCs were first experimentally described as fibroblast-like cells with plastic-adhesive, colony-forming and osteogenic capacities (Friedenstein, 1980). Uniquely, MSCs contribute to bone and other mesenchymal tissue repair either by direct differentiation (Zellner et al., 2014) or indirectly by the release of bioactive mediators that could have paracrine or autocrine activities (Baraniak and McDevitt, 2010; Caplan and Dennis, 2006; Pak et al., 2017). By these mediators, MSCs could support the survival and maturation of end othelial cells and help the formation of new blood vessels through the release of VEGF (Baraniak and McDevitt, 2010; Zwaginga and Doevendans, 2003). Additionally, MSCs produce immunomodulatory factors when stimulated by pro-inflammatory cytokines during the early healing phase (El-Jawhari et al., 2016). This immunomodulation to control inflammation is essential for the effective repair process, particularly osteogenesis (Hoff et al., 2011; Kovach et al., 2015; Lienau et al., 2010).

BM-MSCs, whether detected as colony-forming cells or characterised by specific surface phenotype, e.g. as $\mathrm{CD} 45^{\text {low }} \mathrm{CD} 271^{\text {high }}$ cells, are few in number and constitute less than $0.1 \%$ of the total BM cells (CastroMalaspina et al., 1980; Cuthbert et al., 2012; El-Jawhari et al., 2017; Jones and McGonagle, 2008; Tormin et al., 2011). Furthermore, the number of BM-MSCs varies significantly based on patient age and gender as well as between healthy individuals withing the same age range and gender (Cuthbert et al., 2012; El-Jawhari et al., 2017; Jones and Schafer, 2015; Siegel et al., 2013). In contrast, the culture expansion of BM-MSCs is a costly process with complicated steps for safety and quality control (Capelli et al., 2015). Therefore, $\mathrm{BM}$ concentration has become a popular approach within the practice of various regenerative therapies to enrich MSC quantities and growth factors helping bone and cartilage repair (Hernigou et al., 2005a; Hernigou et al., 2005b; Holton et al., 2016; Moatshe et al., 2017; Zhong et al., 2012). BMAC is considered to be clinically safe being from an autologous source and minimally manipulated (Gobbi et al., 2015; Moatshe et al., 2017; Song and Tuan, 2004). BMAC samples have been used for bone regenerative therapies in cases of complicated fracture healing such as delayed union, non-union, osteonecrosis (especially femoral head necrosis), osseous defects and bone lengthening (Babhulkar et al., 2017; Calori and Giannoudis, 2011; Giannoudis et al., 2013; Giannoudis et al., 2015; Imam et al., 2017; Kanakeshwar et al., 2017; Piuzzi et al., 2017). Additionally, implantation of BMAC has been reported to be a cellular therapy for repair of osteochondral defects, osteoarthritis-related cartilage damage or other chondral defects, mostly combined with supporting scaffolds (Adams et al., 2013; Coppens et al., 2018; Gessmann et al., 2012; Smyth et al., 2012).

The most popular method to achieve BM concentration is the gradient density-based separation of BM components. This separation process involves centrifugation of BM samples and usually takes 1520 min followed by manual removal of the plasma layer and collection of the separated BMAC (or nucleated cells) layer. This process also involves using large-size centrifuges with a swinging horizontal bucket rotor and requires a centrifugation speed up to $1100 \times g$ (El-Jawhari et al., 2017). Heragen ${ }^{\circledR}$ maxx (Heraeus), a recently introduced $\mathrm{BM}$ concentration system, is based on vertical centrifugation. The vertical centrifugation accelerates gravity sedimentation in which a mixture of layers with different densities is allowed to be separated, so that the denser component moves radially and accumulates on the walls of the rotating bowl. In comparison to horizontal centrifugation methods, this vertical centrifugation system concentrates $\mathrm{BM}$ through higher-speed centrifugation, takes a very short time (i.e. $1 \mathrm{~min}$ ) and is designed for an automatic discarding of plasma and RBCs (Douras et al., 2018). However, little is known about the effect of vertical centrifugation on BM-MSC enrichment and whether the fast and high-speed centrifugation has any damaging effect on the cell functions.

The present study aimed to assess BM-MSC yield after vertical centrifugation of BMA when compared to those obtained using gradient density centrifugation. Secondly, the aim was to evaluate the viability and proliferation potential of freshly collected BMACMSCs processed by vertical centrifugation when compared to unprocessed donor-matched MSCs in BMA samples. The culture-expanded BMACderived MSCs were also assessed for differentiation potential and expression of soluble factors involved in angiogenesis and immunomodulation. This 
knowledge could be valuable for further development of BM concentration techniques and potentially for improving bone and cartilage regenerative therapies.

\section{Materials and Methods}

\section{Patient samples}

33 patients (14 males and 19 females; median age: 40 years; range: $21-66$ years) participated in the study. All patients had elective orthopaedic procedures at the Trauma and Orthopaedic unit of Leeds General Infirmary and provided informed written consent according to the ethical approval with NREC number: 06/Q1206/127, National Research Ethics Committee Yorkshire \& Humber-Leeds East. From each patient, $60 \mathrm{~mL}$ of iliac crest BM sample was aspirated by P.V.G. and, then, mixed with anticoagulant citrate dextrose, as previously described (Cuthbert et al., 2015; Hernigou et al., 2013). The samples were loaded into either Heragen maxx ${ }^{\circledR}(12 \times 12 \mathrm{~cm})(n=15)$, BioCUE $^{\text {TM }}(43 \times 37 \times 23 \mathrm{~cm}$, Zimmer-Biomet, Warsaw, IN, USA) $(n=14)$ or SmartPReP-2 ${ }^{\circledR}(46 \times 42 \times 22 \mathrm{~cm}$, Harvest-Tech, Hudson, NY, USA) $(n=4)$ for 1,15 or 14 min centrifugation, respectively. From each of the original BMA and the resulting BMAC samples, $1 \mathrm{~mL}$ paired samples (donor-matched) were collected into EDTA tubes and mixed few times to prevent clotting. These collected samples were transported to the laboratory for experimental processing and analysis within 1-2 h of the collection, as described below.

\section{Processing of BM samples}

The $1 \mathrm{~mL}$ BMA and BMAC samples collected by vertical centrifugation were divided into aliquots for several analytic purposes. $100 \mu \mathrm{L}$ freshly collected samples were used for enumeration of MSCs by flow cytometry. Also, freshly collected BMA and BMAC samples were analysed for counting other BM cells (including RBCs, WBCs and platelets) using an automatic haemocytometer (Sysmex; Sysmex Ltd, Milton Keynes, UK). These samples were diluted 1 : 10 before being processed using the Sysmex. Cell counts were normalised per $1 \mathrm{~mL}$ of BMA or $\mathrm{BMAC}$. The remaining of BMA and BMAC samples were used for CFU-F assays, measuring ROS levels and culture-expansion. BM samples obtained by BioCUE ${ }^{\mathrm{TM}}$ or SmartPReP- $2^{\circledR}$ were only processed for CFU-F assays as controls for colony counts.

\section{Culture-expansion of MSCs}

Paired BMA and BMAC samples were treated with RBC lysis buffer (90 g/L NH${ }_{4} \mathrm{Cl}, 10 \mathrm{~g} / \mathrm{L} \mathrm{KCl}$ and $2 \mathrm{~mL} / \mathrm{L}$ of $0.5 \mathrm{~mol} / \mathrm{L}$ EDTA) and washed twice with PBS (Sigma-Aldrich). The processed cells were distributed in cultures as $2-3 \times 10^{6}$ cells $/ 25 \mathrm{~cm}^{2}$ culture flask (Corning). After $48 \mathrm{~h}$, the medium containing cells in suspension was discarded. Then, plastic-adherent cells were maintained in cultures until 70-80 \% confluency, then they were detached using trypsin, divided $1: 4$ and moved into new flasks (passaged). The medium used for cultureexpansion was StemMACS ${ }^{\mathrm{TM}}$ MSC Expansion Media (Miltenyi Biotec), supplemented with 1 : 100 penicillin/streptomycin (Sigma-Aldrich). Cultures were maintained at $37^{\circ} \mathrm{C}$ and $5 \% \mathrm{CO}_{2}$. The medium was half changed every 3-4 d, and cells were kept in cultures until passage 3. Culture-expanded MSCs were further processed for trilineage differentiation, gene expression assays and soluble mediators' measurements.

\section{CFU-F assay for counting and proliferation of BM- MSCs}

The CFU-F assay was used to enumerate colonyforming cells (MSCs), as reported previously (ElJawhari et al., 2017). Equal volumes $(200 \mu \mathrm{L})$ of paired BMA and BMAC samples were treated with RBC lysis buffer. Then, cells were seeded in $10 \mathrm{~cm}$ culture dish (Corning). Each seeded sample contained an average of $5.3 \times 10^{6}\left(1.2-7.4 \times 10^{6}\right)$ and $21 \times 10^{6}\left(3-38 \times 10^{6}\right)$ cells for BMA and BMAC, respectively. All cell cultures were maintained in StemMACSTM MSC Expansion Media (Miltenyi Biotec), supplemented with 1 : 100 penicillin/streptomycin (Sigma-Aldrich), at $37{ }^{\circ} \mathrm{C}$ and $5 \% \mathrm{CO}_{2}$ for $14 \mathrm{~d}$ with half-medium changes twice weekly. For colony counting, the medium was removed and the colonies were fixed using $10 \%$ formalin (Sigma-Aldrich). Then, methylene blue dye (Sigma-Aldrich) was used to visualise colonies. The colonies were counted, and the normalised count per $1 \mathrm{~mL}$ of BMA or BMAC was calculated. For assessing the proliferative capacity of native MSCs, colonies were scanned and analysed using Image J software (NIH). The coloured RGB images of CFU-F colonies were analysed as binary 8-bit greyscale images and the colony integrated density was calculated as area $\times$ mean grey density value.

\section{Flow cytometry for BM-MSC enumeration}

The method described by El-Jawhari et al. (2017) for the rapid enumeration of BM-MSCs was used. Antibodies against CD271 (Miltenyi Biotec) and CD45 (BD Biosciences), in addition to the live nucleated cell marker Vybrant ${ }^{\circledR}$ DyeCycle ${ }^{\mathrm{TM}}$ Ruby Stain (Thermo Fisher Scientific), were used as recommended by the manufacturers. These antibodies were added into unprocessed freshly collected donor-paired BMA and BMAC samples for $5 \mathrm{~min}$ in the dark at room temperature. The dilutions, volume of antibody/dye : volume of BMA/BMAC sample were $40 \mu \mathrm{L}: 100 \mu \mathrm{L}$ for CD271, $40 \mu \mathrm{L}: 100 \mu \mathrm{L}$ for CD45 and $1 \mu \mathrm{L}: 100 \mu \mathrm{L}$ for Vybrant ${ }^{\circledR}$ DyeCycle $^{\mathrm{TM}}$ Ruby Stain. Data were acquired on Attune NxT Flow Cytometer (Thermo Fisher Scientific). The automated volumetric counts of CD $45^{\text {low }}$ CD271 $1^{\text {high }}$ cells were analysed using Attune NxT Flow Cytometer software. Cell counts were normalised per $1 \mathrm{~mL}$ of BMA or BMAC.

\section{Measuring intracellular ROS levels in BM-MSCs}

A flow-cytometry-based assay was applied to measure intracellular ROS levels in BM-MSCs using 
a fluorogenic oxidative stress sensor, Green CellROX ${ }^{\circledR}$ Flow Cytometry Kit (Thermo Fisher Scientific), as per manufacturer's instructions. After processing with RBC lysis buffer, the freshly collected BM cells were labelled using the surface marker antibodies against CD271 (Miltenyi Biotec) and CD45 (BD Biosciences). Cells were also stained with calcein violet dye (Thermo Fisher Scientific) to define live cells. FMO tube with all antibodies and markers except CellROX ${ }^{\circledR}$ was used to define the positivity of CellROX ${ }^{\circledR}$. Data were acquired on BD ${ }^{\mathrm{TM}}$ LSRII 4 laser flow cytometer and analysed using FACSDiva software (BD Biosciences).

\section{MSC differentiation assays}

The BMA and BMAC culture-expanded MSCs (passage 3) were used for the tri-lineage differentiation assays, as shown previously (El-Jawhari et al., 2019b; Fragkakis et al., 2018). BMA culture-expanded MSCs in the expansion growth medium were used as control, as these BMA cells were the original (standard) population and were not processed by concentration. For the osteogenic assays, MSCs were equally seeded (10,000 cells/well) in the osteogenic StemMACS ${ }^{\mathrm{TM}}$ OsteoDiff Media (Miltenyi Biotec) for 2 weeks before being tested for ALP expression using fast blue stain (Sigma-Aldrich). To visualise the calcium deposition after 3 weeks of osteogenic cultures, alizarin red S stain was used (SigmaAldrich). Furthermore, the calcium levels were quantified using the colorimetric Calcium Liquid kit (Sentinel Diagnostics). Cultured MSCs were lysed in $1 \mathrm{~mol} / \mathrm{L} \mathrm{HCl}$ solution following good mixing, then kept at $-20{ }^{\circ} \mathrm{C}$ until calcium was measured. Absorbance was read at $600 \mathrm{~nm}$ wavelength using Multiskan ${ }^{\circledR}$ EX reader and Ascent ${ }^{\mathrm{TM}}$ software (Thermo Fisher Scientific). Measured calcium levels were normalised to the DNA quantities, which were tested using NanoDrop ${ }^{\mathrm{TM}}$ spectrophotometer (Thermo Fisher Scientific).

For the chondrogenic assays, MSCs were equally distributed (200,000 cells/tube) in the chondrogenic StemMACS ${ }^{\mathrm{TM}}$ ChondroDiff Media (Miltenyi Biotec) in conical shaped tubes to grow as pellets $(200,000$ cells/ pellet). Following 3 weeks of culture, some cell pellets were cryo-fixed and sectioned before being stained for proteoglycans using toluidine blue $\mathrm{O}$ dye (Sigma-Aldrich). Other cell pellets were digested in papain solution $(100 \mathrm{mmol} / \mathrm{L}$ sodium phosphate buffer supplemented with $5 \mathrm{mmol} / \mathrm{L} \mathrm{Na}_{2}$ EDTA, $10 \mathrm{mmol} / \mathrm{L}$ L-cysteine and $100 \mathrm{mg}$ papain in $100 \mathrm{~mL}$ of the prepared buffer, Sigma-Aldrich). Then, the levels of GAG were quantified in papain-lysed cell pellets using a Blyscan ${ }^{\mathrm{TM}}$ kit (Biocolor Life Sciences, Carrickfergus, County Antrim, Ireland).

For the adipogenic assays, MSCs were equally distributed (50,000 cells/well) and cultured in the adipogenic StemMACS ${ }^{\text {TM }}$ AdipoDiff Media (Miltenyi Biotec) in replicate culture-wells. Following 3 weeks of culture, some cultured wells were stained using oil red O (Sigma-Aldrich) for cellular triglycerides and lipids. Other cultured wells were processed for the quantitative fluorescent staining using Nile red (cellular lipid stain, Sigma-Aldrich) and DAPI (nuclei stain, Sigma-Aldrich). The Nile red/DAPI ratio was calculated to assess the Nile red levels normalised to cell counts. All images for differentiated cells were captured using an Eclipse E1000 light microscope (Nikon).

\section{ELISA for soluble factors produced by MSCs}

Culture-expanded BM-MSCs (passage 3) of paired BMA and BMAC samples were seeded equally $(100,000$ cells/well) in 12-well culture plates (Corning). Cells were cultured for $5 \mathrm{~d}$ in the StemMACS ${ }^{\mathrm{TM}}$ MSC Expansion Media. Then, culture supernatants $(1 \mathrm{~mL})$ were collected for analysis of soluble factors. ELISA kits for immunomodulatory factors, PGE2 and TGF $\beta$, as well as for angiogenic factors, VEGFA and angiogenin, were used as per manufacturer's recommendation (Thermo Fisher Scientific). Levels of the tested factors were measured using standard curves and normalised to cell count (measured as DNA quantity using the NanoDrop ${ }^{\mathrm{TM}}$ spectrophotometer).

\section{Gene expression assays}

Passage 3 culture-expanded MSCs (5-10 × 106 cells) from paired BMA and BMAC samples were processed for RNA extraction using a column-based kit (Single Cell RNA Purification Kit; Norgen, Thorold, Canada, ). cDNA synthesis and preamplification steps were conducted using which products? as per manufacturer's instructions (Fluidigm, South San Francisco, CA, USA). Quantitative real-time PCR was performed on Integrated Fluidic Circuits chips (Fluidigm). TaqMan probes were used for tested gene targets (Thermo Fisher Scientific). Probes included SPP1 (Hs00959010_m1), BMP2 (HS00154192_m1), SPARC (Hs00277762_m1), ACAN (Hs00153936_m1), SOX9 (Hs00165814_m1), PPARG (Hs01115513_m1), LEPR (Hs00174492_m1), ANGP1 (Hs00181613_m1), VEGFA (Hs00900058_m1), TGF 31 (Hs00998133_m1), PTGES2 (Hs00228159_m1) and the housekeeping gene HPRT1 (Hs99999909_m1). The relative expression of genes of interest was calculated using the formula: $2^{-}$(Ct of a gene of interest-Ct of HPRTI) (El-Jawhari et al., 2019a).

\section{Statistical analysis}

Figure preparation and statistical analysis were performed using GraphPad Prism 7 software. Paired comparison tests were used for BMA and BMAC samples according to data normality distribution and as indicated in the figure legends. $p<0.05$ was considered statistically significant.

\section{Results}

Vertical-centrifugation-system-enriched BM-MSCs The vertical-centrifugation-based concentration of BMAs was performed as a part of a surgical 
operation and required only 1 min centrifugation. The centrifugation resulted in a separation of three layers of plasma, buffy coat and the rest of BM cells including RBCs (Fig. 1a, step 1). An automatic discarding of other BM cells and RBCs, followed by the plasma layer, was conducted (Fig. 1a, step 2 and 3). Finally, the only remaining BMAC layer was collected using a syringe (Fig. 1a, step 4). The collected buffy coat of BMACs was $6 \mathrm{~mL}$ in all samples and only $1 \mathrm{~mL}$ was used for the laboratory assays. For the enumeration of the freshly collected MSCs, an optimised flowcytometry-based rapid assay was used (El-Jawhari et al., 2017). The flow cytometry plots showed that the numbers of $C D 45^{\text {low }} \mathrm{CD} 271^{\text {high }}$ cells, the surrogates of BM-MSCs, per mL of BMACs were increased when compared to those of donor-matched BMA samples (Fig. 1b). For all samples processed by vertical centrifugation, a significant increase in $C D 45^{\text {low }}$ CD271 ${ }^{\text {high }}$ cell number $/ \mathrm{mL}$ was noted for BMAC $\left(p=0.0001\right.$, Fig. 1c). The number of CD $45^{\text {low }}$ CD271 ${ }^{\text {high }}$ cells was donor-variable as expected (El-Jawhari et al., 2017; Muschler et al., 2001) and ranged between 248 and 62,300 cells (median 1,245 cells/mL) for BMA and
1,605 and 140,000 cells (median 4,470 cells/mL) for BMAC samples. These data indicated a nearly 4-fold enrichment in CD45 ${ }^{\text {low }}$ CD271 1 high cells in BMACs using the vertical centrifugation system.

Both BMAC and BMA cells processed by vertical centrifugation were able to form colonies when seeded in culture but more colonies per volume were observed in the BMAC dishes (Fig. 1d). The number of colonies/mL of BMAC were consistently larger than those of donor-paired BMA samples $(p=0.0312$, Fig. 1e). The number of colonies ranged between 5 and 420 (median 63) for 1 mL of BMA samples and 201,000 (median 270) for $1 \mathrm{~mL}$ of BMAC samples. These data showed a nearly 4.3-fold increase in colonies in BMACs when compared to the same volume of BMA samples.

Age-matched comparison between vertical centrifugation and conventional $\mathrm{BM}$ concentration systems, BioCUE ${ }^{\mathrm{TM}}$ and SmartPReP- ${ }^{\circledR}$ (median age of 37, 40 and 42 years old, respectively), was next performed. Similar to vertical centrifugation data, the colony numbers were increased significantly, 4.6-fold in BMACs using BioCUE ${ }^{\mathrm{TM}}(p=0.0078)$ and 3-fold
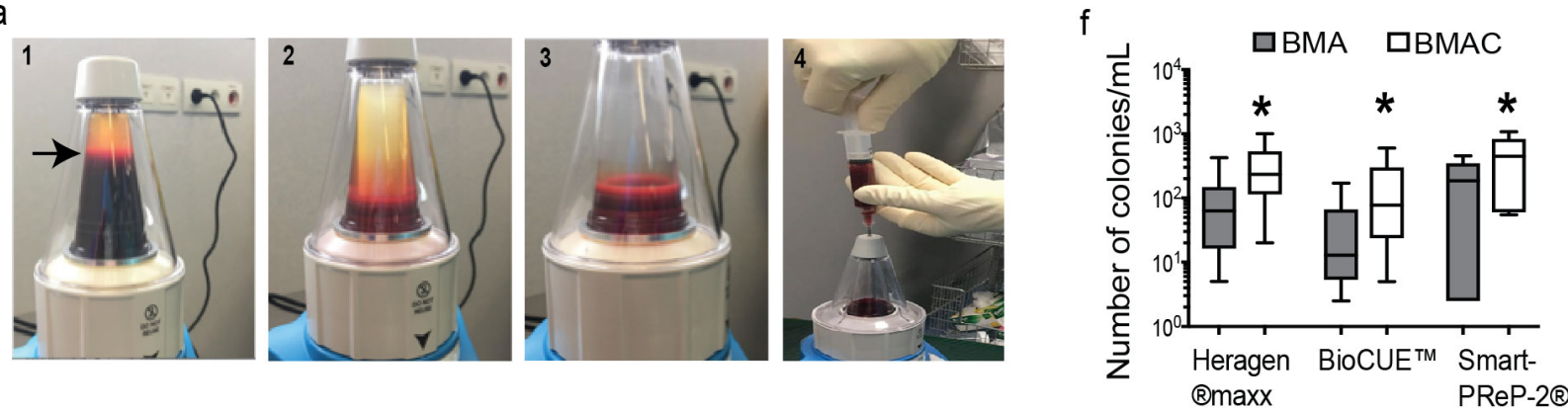

b

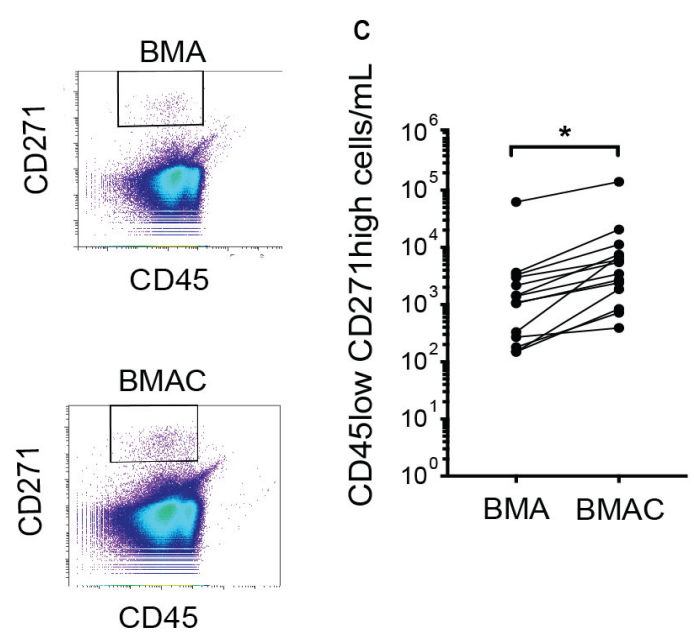

d

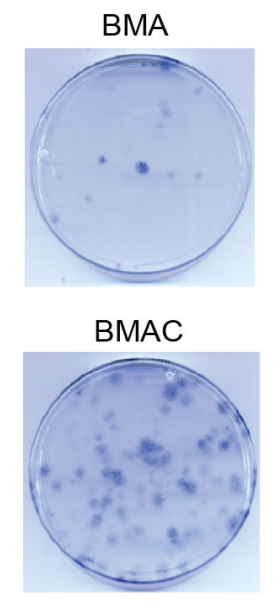

e

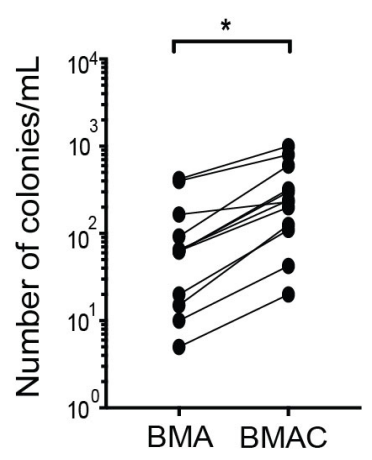

Fig. 1. Enumeration of MSCs in BMAC obtained using the vertical centrifugation system. (a) The vertical centrifugation of BMA, yielding a buffy coat of BMAC (as indicated by the arrow, step 1, 1 min), was followed by the removal of most other BM cells, including RBCs (step 2, few seconds), discarding of plasma (step 3, few seconds) and collection of BMAC into a syringe (step 4). (b) Representative flow cytometry plots showing the gating of the CD45 $5^{\text {low }}$ CD271 $1^{\text {high }}$ cells in BMA and BMAC samples. (c) CD45 ${ }^{\text {low }}$ CD271 ${ }^{\text {high }}$ cell/ $\mathrm{mL}$ of donor-paired BMA and BMAC samples $(n=14)$. (d) Representative CFU-F culture dishes showing the colonies formed by paired BMA and BMAC samples and stained using methylene blue. (e) Number of colonies/mL of paired BMA and BMAC samples $(n=12)$. (f) Number of colonies/mL of BMA and BMAC using vertical centrifugation system (Heragen ${ }^{\circledR}$ maxx), BioCUE ${ }^{\mathrm{TM}}$ and SmartPReP-2 ${ }^{\circledR}(n=10,14$, and 4 respectively). (c,e,f) ${ }^{*} p<0.05$, Wilcoxon matched-pairs signed-rank test. 
for SmartPReP-2 ${ }^{\circledR}(p=0.0156)$ (Fig. 1f). Furthermore, data showed that there was no significant difference between the average colony number/mL of BMAC obtained by the three concentration systems (Fig. 1f).

The main advantage of the tested vertical centrifugation system was the 4-fold enrichment in BM-MSCs in a small volume. This enrichment was comparable to other tested systems for BM concentrations, but importantly with a reduction in processing time from $15-20 \mathrm{~min}$ to $1-2 \mathrm{~min}$.
MSC concentration in relation to other BM cell numbers and donor-related factors

The effect of the vertical centrifugation on the numbers of other BM cells was investigated. Cells were counted using an automatic haematopoietic cell counter, Sysmex. Compared to BMA counts, the numbers of platelets and WBCs per $\mathrm{mL}$ of BMAC were significantly increased (7.6-fold, $p=0.0005$ and 3.7-fold, $p=0.001$, respectively, Fig. $2 \mathbf{a}, \mathbf{b}$ ). In contrast, the number of RBCs per $\mathrm{mL}$ of BMAC was reduced

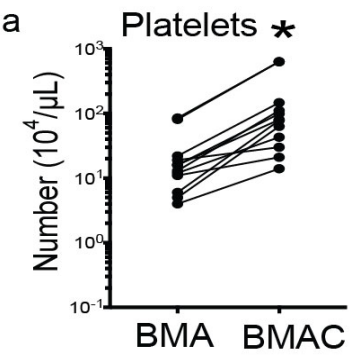

d

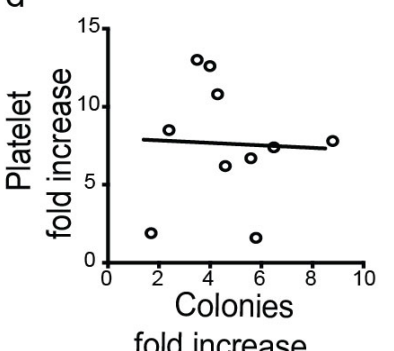

g

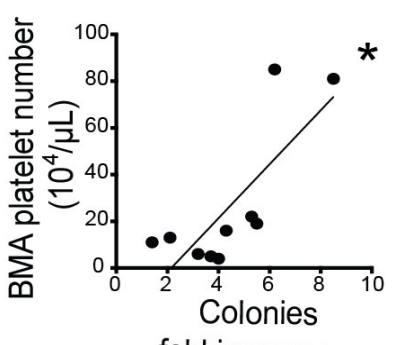

fold increase

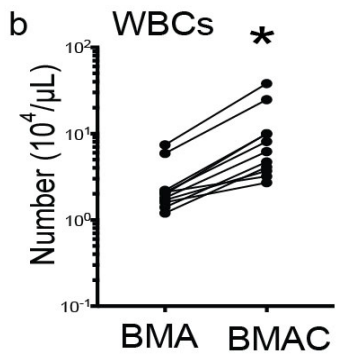

e

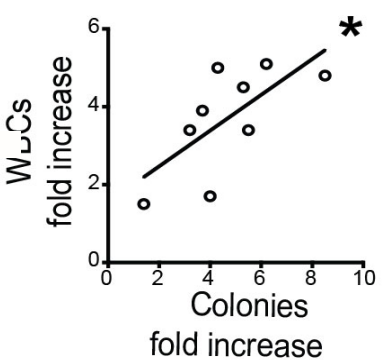

h

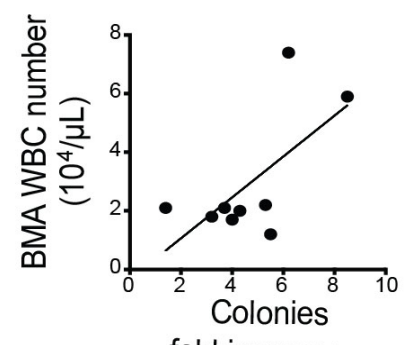

fold increase

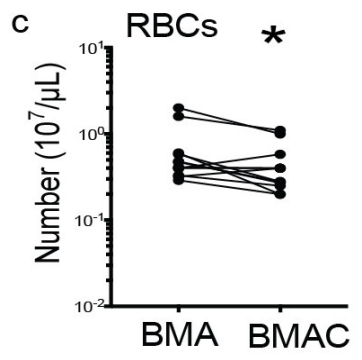

f

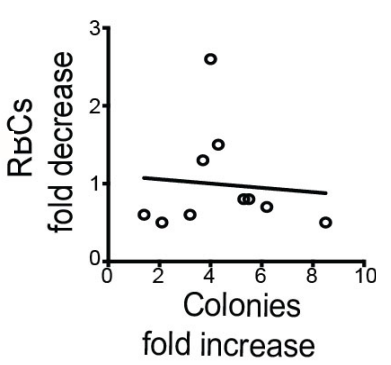

i

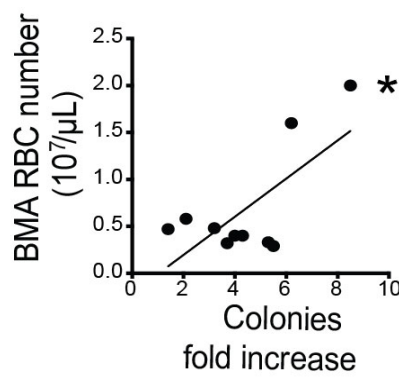

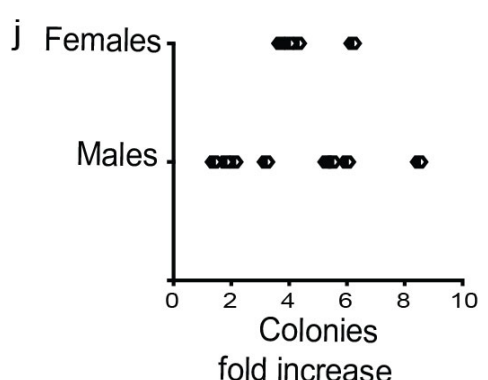

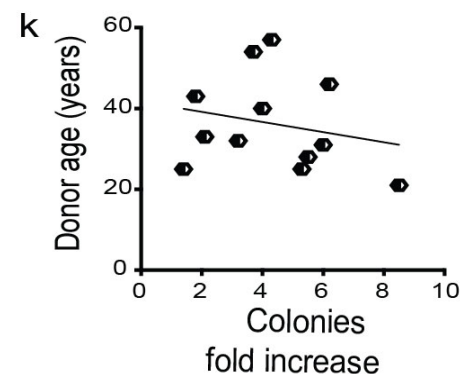

Fig. 2. Various BMA cell counts and donor factors in relation to Heragen ${ }^{\circledR}$ maxx-enriched BM-MSCs. (a-c) Number of platelets, WBCs and RBCs in BMA as compared to BMAC obtained using vertical centrifugation $(n=10)$. (d-f) Fold increase in platelets, WBCs and RBCs versus colony fold increases in BMAC samples processed by vertical centrifugation. (g-i) Number of BMA platelets, WBCs and RBCs versus colony fold increase in BMAC samples processed by vertical centrifugation. (j) Colony fold increase in BMAC samples processed by vertical centrifugation in females versus males. (k) Donor age versus fold colony increase in BMAC samples processed by vertical centrifugation. ${ }^{*} p<0.05$. (a-c) Wilcoxon matched-pairs signed-rank test was used for paired comparisons. (d-k) Spearman's rho test was applied. 
considerably as compared to that of BMA (25\% reduction, $p=0.0205$, Fig. $2 c$ ). These data indicated an enrichment in platelets and WBCs as well as a loss of RBCs in BMAC processed by vertical centrifugation.

Next, the relationships between the enrichment of BM-MSCs and changes in other BM cells following concentration were studied. Interestingly, the colony fold increases positively correlated with the fold increase of WBCs $(p=0.0415$, Fig. 2e). However, the colony fold increases were not statistically correlated with the increases in platelets or the decreases in RBCs (Fig. 2d,f). With regards to the cell counts in BMA, the fold increases in colony numbers (enrichment) were significantly and positively correlated with the counts of platelets and RBCs in BMA ( $p=0.0076$ and $p=0.0245$, respectively, Fig. 2 g,i). Furthermore, there was a positive correlation trend between the colony fold increase (enrichment) and the number of WBCs in BMA, despite not being statistically significant (Fig. 2h).

Looking at other donor-related factors that might have influenced the enrichment of BM-MSCs, male and female donors had similar median levels of 4-fold in colony fold increases (enrichment) (Fig. $2 j$ ). Additionally, the donor age did not show any correlation with the fold increase in colony number (Fig. 2k).

Taken together, another key advantage of BM concentration by vertical centrifugation appeared to be the enrichment of WBCs and platelets. No linkage to age or gender was observed. a

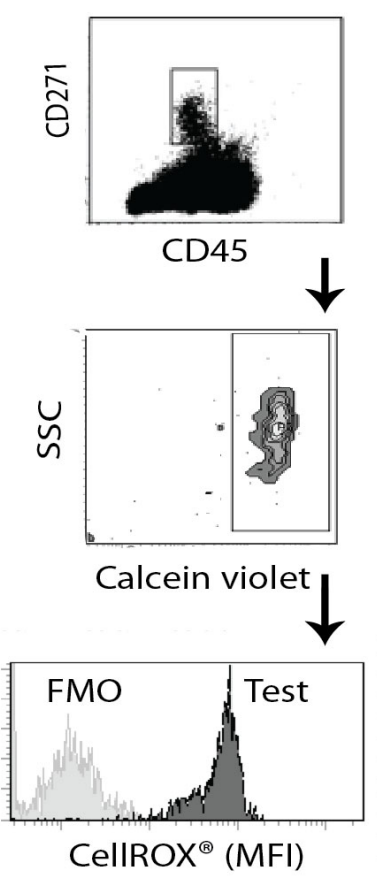

d

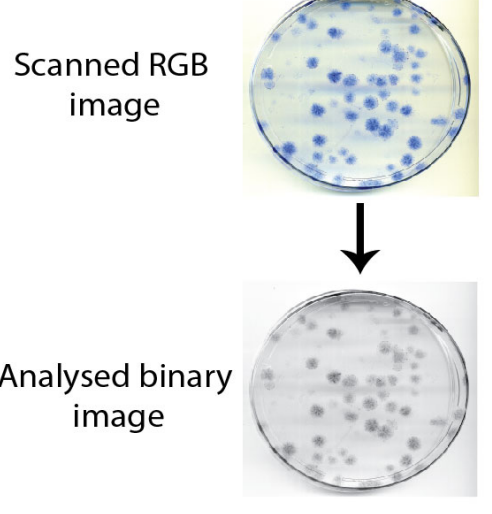

b

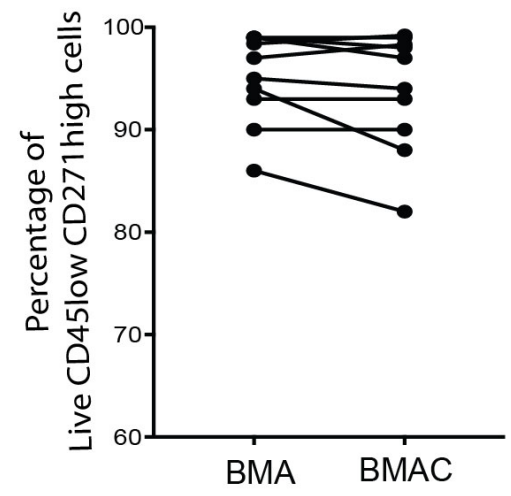

c

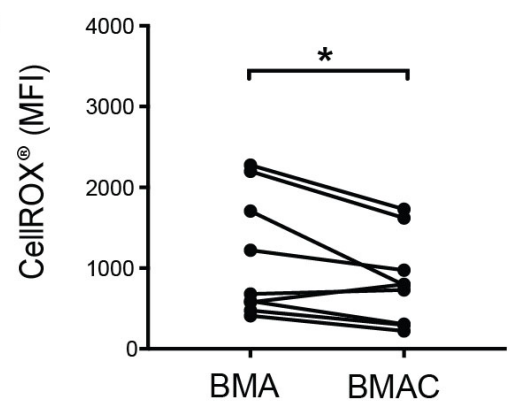

e

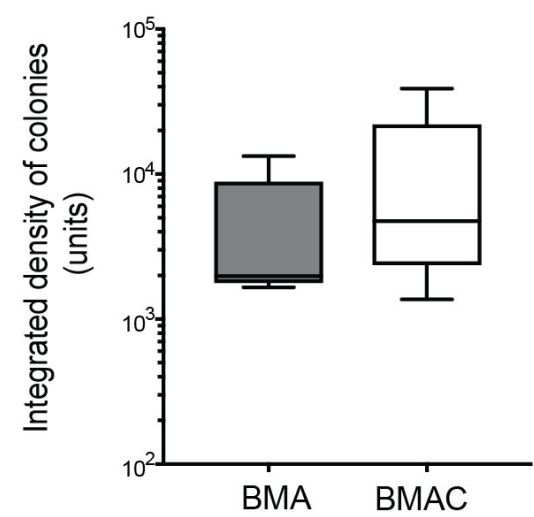

Fig. 3. Survival, ROS levels and proliferation of BM-MSCs after using the vertical centrifugation system. (a) Flow cytometry gating on $\mathrm{CD} 45^{\text {low }} \mathrm{CD} 271^{\text {high }}$ cells. Live cells were identified as calcein violet positive, followed by CellROX ${ }^{\circledR}$ MFI measurement in a test (black histogram) or Fluorescence Minus One Control FMO (grey histogram). (b) Percentage of live CD45 ${ }^{\text {low }}$ CD271 ${ }^{\text {high }}$ cells in BMA and BMAC samples. (c) CellROX ${ }^{\circledR}$ levels were measured in paired $C D 45^{\text {low }}$ CD271 ${ }^{\text {high }}$ cells in BMA and BMAC samples $(n=9)$. (d) Scanned RGB colonies were analysed using image J software as binary image for integrated density. (e) Median of integrated density units between BMA and BMAC colonies ( $n=5$ donors, Mann-Whitney U test). (b,c,e) ${ }^{*} p<0.05$, paired $t$-test was used for paired comparisons. 
MSCs processed by vertical centrifugation did not show an induction of intracellular ROS and maintained their survival and proliferative capacity Quantity, stress status, survival and proliferation potential of MSCs following BM concentration by vertical centrifugation were assessed. By flow cytometry, the survival of CD $45^{\text {low }} \mathrm{CD} 271^{\text {high }}$ cells was analysed using calcein violet dye (Fig. 3a). Then, these live cells were further analysed for intracellular ROS levels by measuring the MFI levels of CellROX ${ }^{\circledR}$ dye expression (Fig. 3a). The percentage of live CD45 ${ }^{\text {low }}$ CD271 ${ }^{\text {high }}$ cells was comparable for both BMA and BMAC samples (median of $96 \%$ and $95 \%$, respectively; Fig. 3b). Interestingly, the levels of intracellular ROS in BMAC samples were not induced but appeared to be lower than those of BMA samples $(p=0.0323$, Fig. 3c)

The colonies formed in BMA and BMAC cultures were analysed for their density to assess the proliferative potential of MSCs (Fig. 3d). Image a

Fast blue

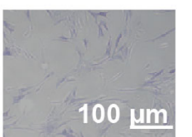

BMA

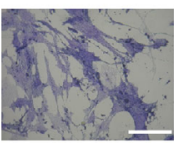

BMAC
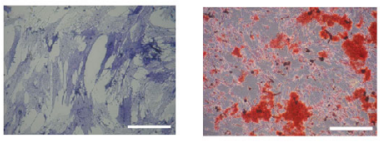

b
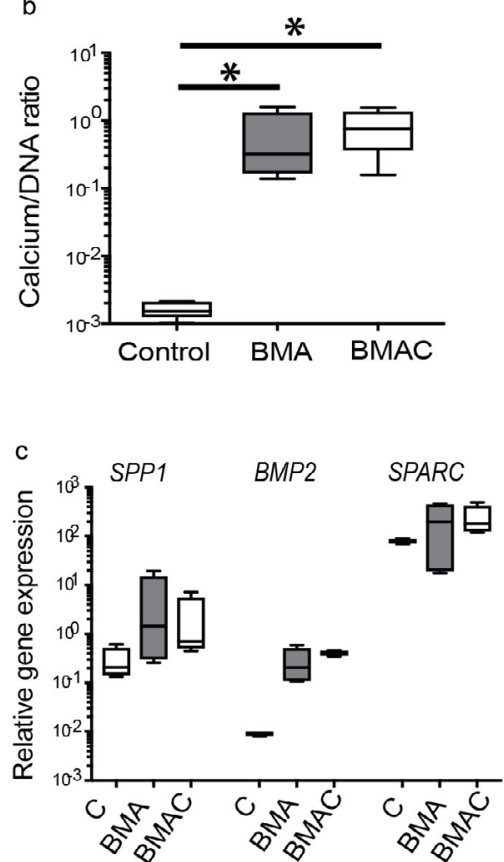

d

Toluidine blue

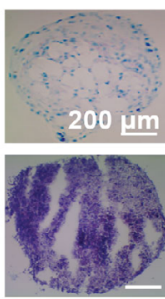

BMAC
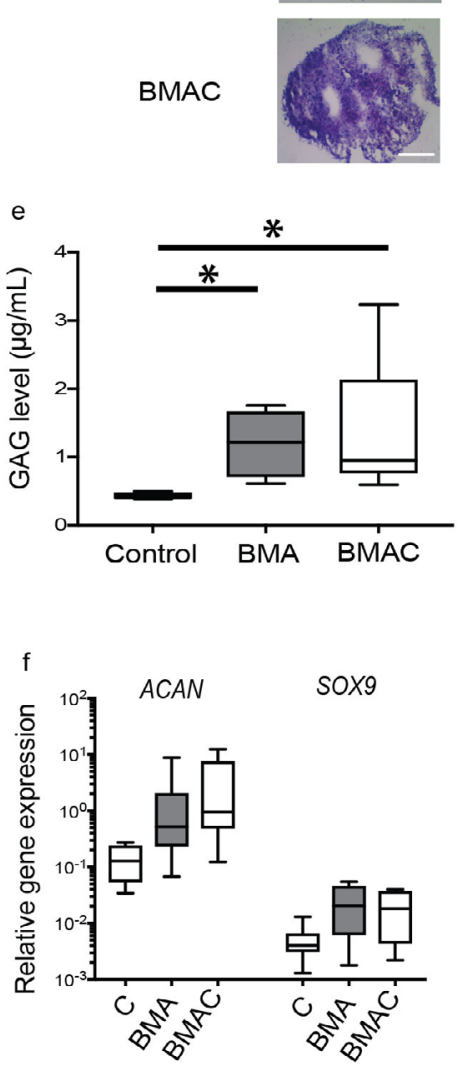

Control expansion

BMA g

BMA
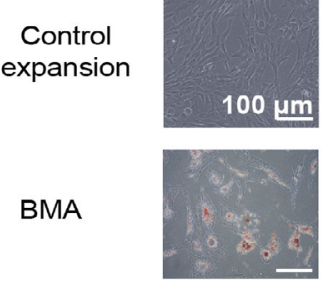

BMAC
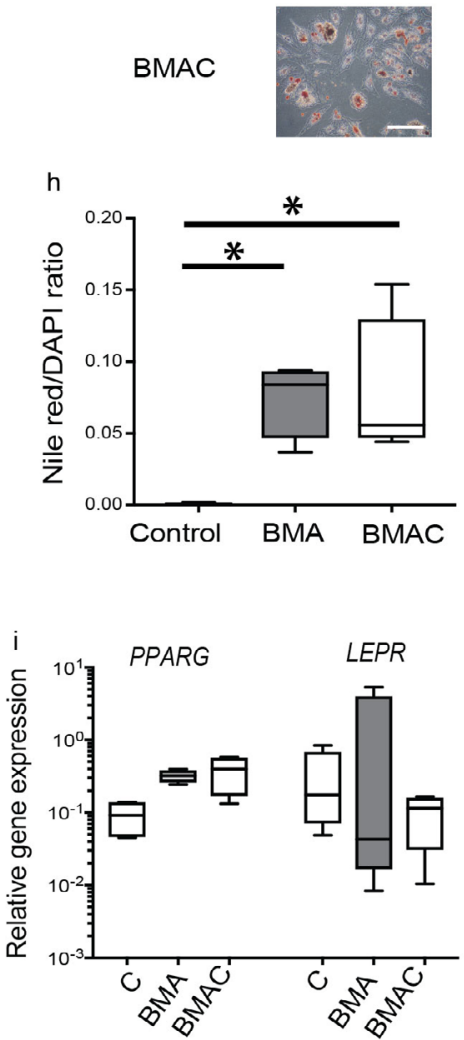

Fig. 4. Differentiation of culture-expanded BM-MSCs after vertical centrifugation processing. (a) Fast blue and alizarin red stainings for BMA- and BMAC-MSCs after culture in osteogenic medium for 2 weeks (for fast blue) or 3 weeks (for alizarin red). Control images for BMA-MSCs in expansion medium are shown. (b) Levels of calcium normalised to cell counts of BMA- and BMAC-MSCs maintained in osteogenic medium for 3 weeks $(n=5)$ and control BMA-MSCs (expansion, $n=5) .{ }^{*} p<0.05$. (c) Relative gene expression levels of osteogenic markers $(n=4)$. Control: BMA-MSCs (day $0, n=4)$. (d) Toluidine blue staining for BMA- and BMAC-MSC pellets maintained in chondrogenic medium for 3 weeks. A control image for BMA-MSC pellet in expansion medium is shown. (e) GAG levels in BMA- and BMAC-MSCs maintained in chondrogenic medium for 3 weeks $(n=5)$ and control BMA-MSCs (expansion, $n=5) .{ }^{*} p<0.05$. (f) Relative gene expression levels of chondrogenic markers $(n=4)$. Control: BMA-MSCs (day 0, $n=4)$. (g) Oil red O staining for BMA- and BMAC-MSCs maintained in adipogenic medium for 3 weeks. A control image for BMA-MSCs in expansion medium is shown. (h) Nile red/DAPI ratios for BMA- and BMAC-MSCs maintained in adipogenic medium for 3 weeks $(n=5)$ and control BMA-MSCs (expansion, $n=4) .{ }^{*} p<0.05$. (i) Relative gene expression levels of adipogenic markers $(n=4)$. Control: BMA-MSCs (day $0, n=4)$. Wilcoxon matched-pairs signed-rank test was used for group comparisons except for $(\mathbf{b})$ calcium levels, where paired $t$-test was used. 
J semi-quantitative analysis showed that there was no significant difference in the colony integrated densities between donor-matched BMA and BMAC samples $(p=0.312$, Fig. 3e), suggesting preservation of the proliferative capacity of MSCs.

In summary, there was no significant difference in BMAC versus BMA fraction in terms of ROS induction using vertical centrifugation for $\mathrm{BM}$ concentration. Furthermore, the survival and proliferative capacities of BM-MSCs after processing were maintained.

MSCs processed by vertical centrifugation showed preserved tri-lineage differentiation potential

To assess the repair functions of MSCs processed by vertical centrifugation, culture-expanded BMAand BMAC-MSCs were investigated using the mesenchymal lineage differentiation assays. The osteogenic cultures showed that both BMA- and BMAC-MSCs were positively stained when using fast blue more than expansion controls, indicating induction of ALP expression in differentiating MSCs (Fig. 4a, left panel). Furthermore, BMA- and BMAC-MSCs in osteogenic cultures were similarly positively stained for alizarin red, indicating effective deposition of calcium (Fig. 4a, right panel). Calcium quantitative measurement (normalised to cell counts) demonstrated a significant increase when compared to control ( $p=0.0079)$ but comparable levels between the two populations, BMA- and BMAC-MSCs, with no statistically significant difference $(p=0.5919$, Fig. 4 b). These data were further confirmed by comparable gene expression levels of induced osteogenic markers (compared to control): SPP1, $B M P 2$ and SPARC $(p=0.2656, p=0.1484$ and $p>0.999$, respectively, Fig. 4c).

After a 3 week culture in the chondrogenic medium, BMA and BMAC MSC pellets were positively stained with toluidine dye, differently from control MSCs in expansion medium, indicating successful chondrogenic differentiation (Fig. 4d). The quantitative levels of GAGs in BMA- and BMACMSCs were found to be significantly higher than in the control $(p=0.0159)$ but to a similar extent in the two populations (median of 1.2 and $1 \mu \mathrm{g} /$ $\mathrm{mL}$, respectively), with no significant difference $(p=0.8125$, Fig. 4e). Furthermore, the gene expression levels of the chondrogenic markers ACAN and SOX9 were higher in differentiating BMA- and BMACMSCs than in control, but no significant difference was found between BMA and BMAC $(p=0.1641$ and $p=0.7188$, Fig. 4 f).

With regards to the adipogenic assay, both BMAand BMAC-MSCs adipogenic cultures demonstrated red staining when using oil red $\mathrm{O}$ dye as compared to control cultures in expansion medium, suggesting the presence of lipid droplets (Fig. $4 \mathrm{~g}$ ). When these adipogenic induced MSCs were analysed for Nile red and DAPI fluorescence, the values of Nile red/DAPI ratio were also similar for differentiating BMA- and BMAC-MSCs (higer, relative to control, $p=0.0159$ ), with no significant difference between the two populations ( $p=0.9080$, Fig. $4 \mathrm{~h})$. Additionally, the gene expression levels for the adipogenic markers PPARG and LEPR were comparable for both MSCs $(p=0.0625, p>0.999$, respectively, Fig. $4 \mathbf{i})$.

Collectively, analysis of trilineage potential showed no apparent differences between BMA and BMAC populations using the vertical centrifugation system, given the following results:

i) osteogenesis, comparable and no significant differences in gene expression levels for SPP1, BMP2 and SPARC;

ii) chondrogenesis, comparable and no significant differences in gene expression levels for $A C A N$ and SOX9;

iii) adipogenesis, comparable and no significant differences in gene expression levels for PPARG and LEPR.

\section{MSCs processed by vertical centrifugation maintained angiogenic and immunomodulatory potential}

Whether the soluble factors released by the BMACMSCs were affected by the vertical centrifugation was tested. The culture supernatants from paired BMA and BMAC culture-expanded MSCs were analysed for levels of MSC-derived soluble factors involved in the immunomodulatory and vascular support activity. The levels of angiogenin and VEGFA in the culture supernatants with or without cell normalisation were similar for donor-paired BMA- and BMAC-MSCs, with no statistically significant difference $(p=0.7109$ and $p=0.9375$, respectively, Fig. 5a). Furthermore, PGE2 and TGF $\beta-1$ levels in the culture supernatants, with or without normalisation to cell counts, were similar for donor-paired BMA- and BMAC-MSCs $(p=0.1562$ and $p=0.1484$, respectively, Fig. $5 b)$.

To further confirm the similar levels of angiogenic and immunomodulatory soluble mediators, the gene expression levels of these markers were tested. Likewise, the transcripts for ANGP1 and VEGFA were comparable between BMA- and BMACMSCs ( $p=0.7109$ for both, Fig. 5c). Also, there was no statistically significant difference in transcript expression levels of TGF $\beta$ - 1 and PTGES2 between BMA- and BMAC-MSCs $(p=0.1484$, and $p=0.7109$, respectively, Fig. 5d).

In summary, ANGP1 and VEGFA expression was not significantly different between BMA- and BMACMSCs. Similarly, immunomodulatory potential was not significantly different between BMA- and BMACMSCs, as similar expression levels of TGF $\beta-1$ and PTGES2 were found.

\section{Discussion}

The present study uniquely tested a vertical centrifugation system, a fast and new technique for BM concentration. The main advantage of this 
vertical centrifugation system was obtaining a 4-fold enrichment in CFU-F colonies (MSCs) per volume of BMAC. Another advantage was the reduction of the typical centrifugation processing time from 15-20 min to 1-2 min, with the enrichment of WBCs and platelets. Additionally, the trilineage multipotentiality, immunomodulatory and angiogenic properties examined for BMA- and BMAC-MSCs were equivalent, with no significant difference. The comparable and non-significant differences in properties between BMA- and BMAC-MSCs indicated that the high-speed vertical centrifugation was not detrimental to BMAC cell functions. Furthermore, as these cells were tested for differentiation and immunomodulatory potential after expansion, this also suggested their suitability for various therapeutic uses. In addition to the CFU-F assay, an optimised and published method was utilised to obtain BM-MSCs surrogates with surface phenotype CD45 ${ }^{\text {low }}$ CD271 $1^{\text {high }}$ (El-Jawhari et al., 2017), which specifically have significant osteogenic differentiation ability (Churchman et al.,
2012, Cuthbert et al., 2012). Both quantitative CFU-F and flow-cytometry methods showed a significant increase in MSC numbers (4-fold) per mL of BMAC relative to the same volume of BMA. Although the CFU-F assay is the standard method for BM-MSC counting, this assay cannot provide timely results as it requires several days in culture. The seeding for the CFU-F assay was performed based on equal volumes of the samples (BMA or BMAC). However, the numbers of colonies per volume were variable between donors as expected, probably related to age and gender, as reported before (El-Jawhari et al., 2017; Muschler et al., 2001). Therefore, the optimised flow cytometry method described in the present study (that takes up to $20 \mathrm{~min}$ ) could potentially help to evaluate BMAC quality (MSC content) within intraoperative clinical settings.

The vertical centrifugation system for BM concentration also showed a comparable enrichment of MSCs (4-fold) with two other tested horizontal centrifugation systems, BioCUE ${ }^{\mathrm{TM}}$ (4.6-fold) and SmartPReP-2 ${ }^{\circledR}$ (3-fold). Additionally, Emcyte
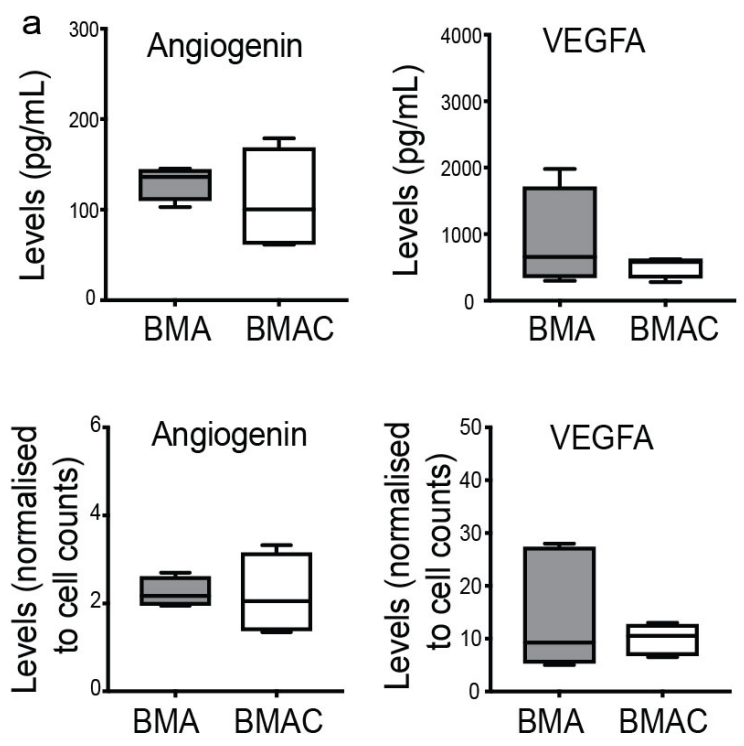

C

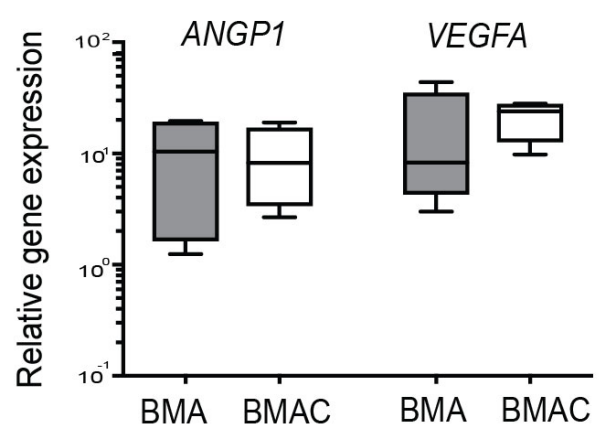

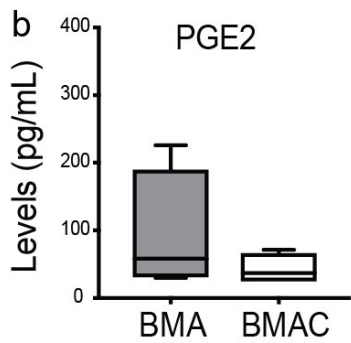
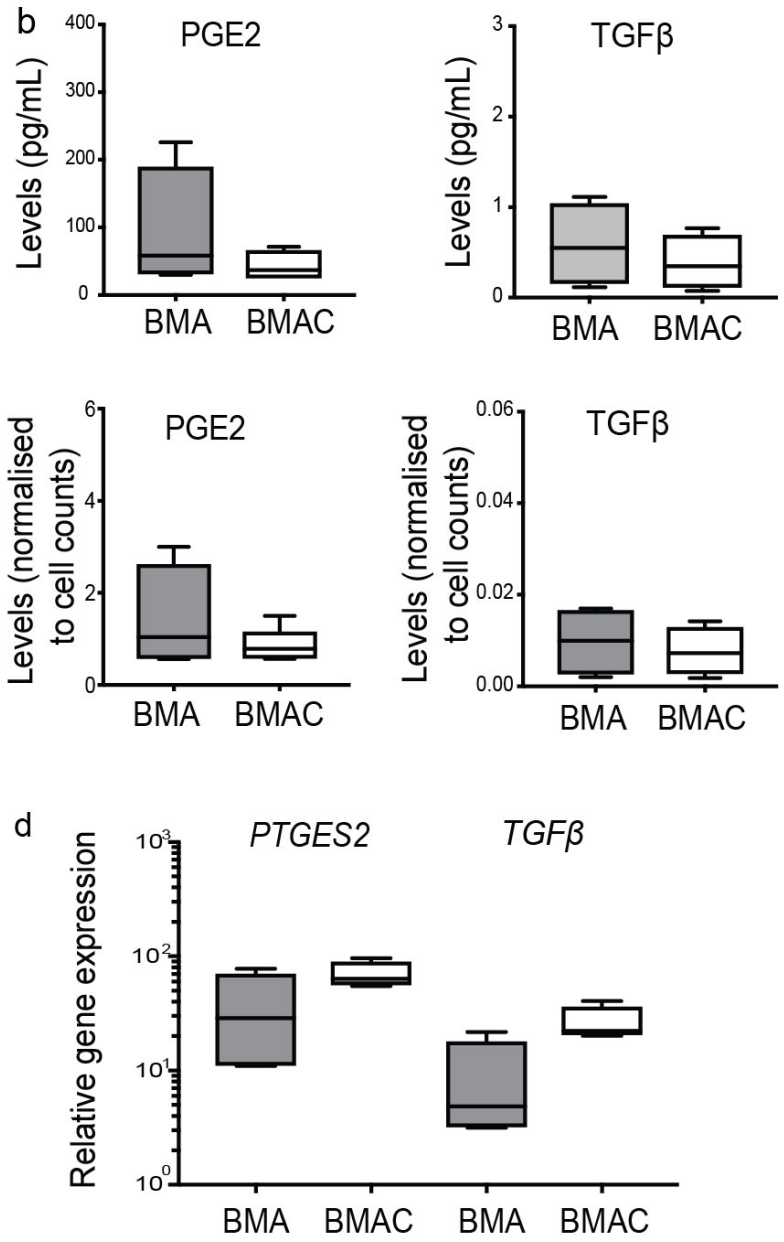

Fig. 5. Soluble factors produced by vertical-centrifugation-processed BM-MSCs. (a) Total (pg/mL) or normalised to cell count levels of angiogenic mediators in the supernatants of culture-expanded MSCs derived from BMA and BMAC $(n=4)$. (b) Total $(\mathrm{pg} / \mathrm{mL})$ or normalised to cell count levels of immunomodulatory-related mediators in the supernatants of culture-expanded MSCs derived from BMA and BMAC $(n=4)$. (c) Relative gene expression levels of angiogenic mediators $(n=4)$. (d) Relative gene expression levels of immunomodulatory mediators $(n=4)$. Wilcoxon matched-pairs signed-rank test was used for group comparisons. 
GenesisCS ${ }^{\circledR}$ (Emcyte, Fort Myers, FL, USA) and Harvest-Tech systems have been shown to enrich MSCs (as CD45 low CD271 high cells) significantly (Schafer et al., 2019), further confirming comparable performance for these systems. It was not feasible to get samples from the same donor for three systems and ideally to perform a paired comparison of BM-MSCs. However, an age-matched comparison was conducted between the tested systems and the colony enrichment were similar. Furthermore, both vertical and horizontal centrifugation systems helped to reduce RBCs and increase the number of platelets and WBCs per volume of BMAC. Horizontal centrifugation systems such as BioCUETM and SmartPReP- $2^{\circledR}$ need large-sized equipment (i.e. centrifuge) and usually need 15 min centrifugation time (El-Jawhari et al., 2017; Schafer et al., 2019). However, smaller centrifuge/device size and shorter centrifugation time (1 min) for the studied system suggested that vertical centrifugation could be a better tool for BM concentration. Additionally, the easy handling of the sample is another advantage of this system because the unused BM components (plasma and many RBCs) can be removed within seconds by pressing a button, leaving only BMAC buffy coat. Additionally, BMAC buffy coat can easily be extracted from the disposable device using a syringe.

An enrichment in platelet and WBC counts was detected (7.6- and 3.7-fold, respectively) as well as a $25 \%$ reduction in RBCs in BMAC following vertical centrifugation. The platelet enrichment is highly valued as these cells contain various growth factors that could enhance osteogenesis, chondrogenesis and angiogenesis during repair (Altaie et al., 2016; Cassano et al., 2018). Furthermore, PRP and BMAC are increasingly investigated in the laboratory or clinical settings as a combined product for regenerative therapies, particularly for bone repair (Betsch et al., 2013; Lee et al., 2014; Smyth et al., 2012). The enrichment in WBCs could be of value in regenerative therapies as MSC and WBC interactions have been shown to be involved in bone and other tissue healing (El-Jawhari et al., 2016). Also, an interesting positive correlation between MSC and WBC enrichment was found, probably because MSCs and WBCs were co-isolated in the buffy coat layer following processing. Further investigations of the biological significance of WBCs in BMAC preparation for tissue repair and how this effect could be correlated with clinical efficacy of treatment are needed. A positive and significant correlation between high cellularity of BMA (particularly platelets and RBCs) and MSC enrichment was additionally noted. This finding could indicate that the larger the BMA cell number, the more efficient is the BM concentration. This observation agreed with a previous report indicating that the cellularity of a buffy coat collected by BM centrifugation correlates well with original BM cellularity (Chang et al., 2010). Also, the present study finding could suggest a probable risk of a reduced MSC yield after concentration in conditions associated with decrease BM cellularity, e.g. ageing.

$\mathrm{BM}$ processing by vertical centrifugation may reduce cell viability or increase stress levels in these cells (Takeshima et al., 2017). However, the viability of BMAC-MSCs was maintained after vertical centrifugation. In agreement, a recent study reported that cell viability is nearly $90 \%$ after processing of BMA by two other concentration systems, Emcyte GenesisCS ${ }^{\circledR}$ and Harvest-Tech devices (Schafer et al., 2019). Interestingly, ROS levels were found to be reduced in BMAC processed by vertical centrifugation when compared to BMA. These data may indicate a cellular response to low oxygen during processing since hypoxia can reduce ROS levels (Kucera et al., 2017). Additionally, ROS levels have been suggested to have a regulating functions in normal cell responses (i.e. redox biology) rather than being linked to cell damage (Schieber and Chandel, 2014). Despite the noted reduction in ROS levels following processing by vertical centrifugation, using semi-quantitative assay (colony integrated density), native BMAC-MSCs were able to form colonies with similar proliferative potential to the donor-matched unprocessed BMA-MSCs.

In addition to the quantity, the quality of BMACMSCs is also important to consider when assessing the effectiveness of BM concentration systems. For BioCUE $^{\mathrm{TM}}$, BMAC-MSCs isolated using this system have competent CFU-F formation as well as ALP expression in osteogenic medium (Hakimi et al., 2014; Woodell-May et al., 2015). Additionally, an in vivo study has proven the osteogenic capacity of these cells (Hakimi et al., 2014). Moreover, BMACMSCs from samples processed by SmartPReP- $2^{\circledR}$ effectively secrete angiogenic factors and express ALP, indicating preserved osteogenic capability (Jager et al., 2011; Schafer et al., 2019). Uniquely, the in vitro assays performed in the present study aimed to assess the differentiation capabilities of BMAC-MSCs derived from vertical-centrifugationprocessed samples as compared to those of donormatched unprocessed BMA samples. Using both qualitative and quantitative methods, the present study showed for the first time that human cultureexpanded BMAC-MSCs from vertical centrifugation displayed active osteogenesis, chondrogenesis and adipogenesis. The secretion of immunomodulatory and vascular mediators by these BMAC-MSCs appeared intact, as indicated by the soluble factor levels in cultures and their gene expression levels. The angiogenic functions of MSCs are mainly related to VEGF, which modulates migration and mitosis of endothelial cells, thus helping to initiate angiogenesis in response to tissue injuries (Ferrara and Gerber, 2001; Holmes et al., 2007). Additionally, immunomodulatory mediators such as TGF $\beta$ and PGE2 have been linked to the role of MSCs in controlling inflammation associated with tissue damage, which could help the progress of tissue repair, particularly bone and cartilage (El-Jawhari et 
al., 2016). Although no functional assays for Heragen ${ }^{\circledR}$ maxx-processed BMAC cells were performed, the present study assessed, for the first time, the secretion of these angiogenic and immunomodulatory factors by MSCs processed by vertical centrifugation. A previous study has shown that the total levels of soluble factors such as platelet-derived growth factor-BB, VEGF, macrophage colony-stimulating factor and interleukin-1 are preserved following $\mathrm{BM}$ concentration using horizontal systems such as SmartPReP-2 ${ }^{\circledR}$ (Schafer et al., 2019).

The preserved differentiation functionality and angiogenic potential of BMAC-MSCs processed by vertical centrifugation indicated their high potential value for mesenchymal tissue regeneration. Concentrated BM-MSCs have promising reparative effects in various pathological conditions, such as degenerative osteochondral lesions, osteoarthritisassociated cartilage loss and bone fractures. Both animal studies and human clinical trials have reported positive results for BMAC application in bone and cartilage healing (Connolly et al., 1989; Zhong et al., 2012). Also, Gobbi et al. (2011; 2015) demonstrated that patients with large patellofemoral chondral lesions show significantly improved clinical outcomes after autologous application with BMAC gained by Harvest-Tech systems, with no adverse effects. Furthermore, conventional centrifugation yielding BMAC is valuable to treat atrophic non-union of tibial fractures (Hernigou et al., 2005a; 2005b). With regards to vertical centrifugation, Douras et al. (2018) have recently reported successful healing of a medial malleus non-union fracture case using this system. In this case report, an application of BMAC together with a BMP-2 sponge and iliac crest autologous graft, according to the elements of 'diamond concept' for bone regeneration (Giannoudis et al., 2007), was associated with a gradual integration of the bone graft implanted and a complete osseous healing at the 6 months follow up (Douras et al., 2018). In addition to regeneration, BMAC-MSCs processed by vertical centrifugation could maintain the production of immunomodulatory factors; thus, these cells can be potentially valuable in treating inflammatory/ autoimmune-based conditions. Examples of MSC application due to their immunomodulatory capacity include graft-versus-host disease, asthma, ulcerative colitis, multiple sclerosis and systemic lupus erythematosus (Gao et al., 2016). More studies would be needed to assess the efficacy of BMAC produced by vertical centrifugation in various regenerative and inflammatory conditions and within large cohorts of patients.

Different surface markers including CD271 have been proposed to characterise BM-MSCs (Boxall and Jones, 2012; 2015). Furthermore, BM-MSCs have also been suggested to contain different subsets of cells according to the expression of other surface markers (Elahi et al., 2016). This heterogeneity of BM-MSC phenotypes may as well be exacerbated by the donor to donor variation. One limitation of the present study could be that the biology of native BMMSCs was assessed as CD45 ${ }^{\text {low }} \mathrm{CD} 271^{\text {high }}$; however, this population is considered to be a surrogate population of BM-MSCs (El-Jawhari et al., 2017). Furthermore, CFU-F assays was also used in addition to the validated flow cytometry phenotype and both methods consistently showed enrichment of MSCs and maintenance of their survival and proliferative capacities. It would be interesting in future work to use antibodies from different suppliers to characterise the MSC populations. For differentiation assays and soluble mediator levels, culture-expanded MSCs, as standardly defined in vitro, were used (Dominici et al., 2006) to gain adequate numbers of these cells for these functional assays. The osteogenesis and adipogenesis assay outcomes were also normalised to cell counts. Although the GAG assay was performed without cell normalisation, the chondrogenic marker expression was quantitively measured relative to a housekeeping gene to show the cellular levels. Future testing of a wider range of tri-lineage markers and assays would aid interpretation of the MSC fractions isolated. Another limitation of the study was that it only analysed the regenerative capacity of BMACderived cells in vitro, and future in vivo studies will be interesting to confirm the value of such BMconcentrating system.

\section{Conclusions}

The process of centrifugation-based $\mathrm{BM}$ concentration helps to enrich MSCs in a small volume of BMAC, which is valuable for small sized bone defects and loading scaffolds or membranes used for various bone/cartilage regenerative therapies. Other methods for separating or enriching BM-MSCs, such as antibody-biding methods, FACS and MACS are also potentially useful (Tomlinson et al., 2013). However, compared to centrifugation systems, these antibodybased techniques need more time and include extra manipulation steps with the use of chemical reagents. Of note, applying recommended BM aspiration techniques, such as using small syringes for collection and multiple aspirates, could be essential to avoid the dilution of BMA samples and to improve the yield of BM-MSCs regardless of the enrichment method (Hernigou et al., 2013). The tested vertical centrifugation system for BM concentration was as effective in enriching MSCs in a small volume and more importantly in a much shorter time than conventional horizontal centrifugation systems. Additionally, this vertical centrifugation system has other more practical advantages, including the compact size of the instrument and the ease to collect BMAC layer as well as discard plasma and many RBCs. A limitation of the study was that the functionality of the BMAC-MSCs was not analysed across the three BM concentration devices. However, vertical centrifugation did not compromise the functional potentials of BMAC-MSCs as compared to 
donor-matched unprocessed BMA-MSCs. The vertical centrifugation system helped also to enrich platelets, which have great potential to enhance bone and cartilage regeneration. Such a fast BM concentration system could potentially be a helpful tool to optimise successful bone and cartilage regenerative therapies. For future work, it will be interesting to assess the in vivo functions of these BMAC-MSCs in animal models after using the vertical centrifugation system in comparison to the other BM concentration methods. Such investigation would aid determination of the effectiveness of these BM systems for wider research application. Moreover, it would be valuable to investigate if and how the numbers of applied MSCs together with other BMAC cells (e.g. WBCs and platelets) are correlated with improved clinical efficacy for different tissue regenerative applications.

\section{Acknowledgments}

We would like to thank the staff of the Academic unit of Trauma and Orthopaedic Surgery, Leeds General Infirmary for their kind help with the sample collection. This research was funded by Heraeus, grant number RG. RMEX.114350. J.J.E. was also funded by a start-up grant from the AO Foundation (number, S-16-132E). D.C.I. was funded by a 110 University of Leeds PhD scholarship. W.J. was funded by EPSRC Centre for Doctoral Training in Tissue Engineering and Regenerative Medicine grant (EP/ L014823/1).

\section{References}

Adams SB, Lewis JS Jr, Gupta AK, Parekh SG, Miller SD, Schon LC (2013) Cannulated screw delivery of bone marrow aspirate concentrate to a stress fracture nonunion: technique tip. Foot Ankle Int 34: 740-744.

Altaie A, Owston H, Jones E (2016) Use of platelet lysate for bone regeneration - are we ready for clinical translation? World J Stem Cells 8: 47-55.

Babhulkar S, Babhulkar S, Vasudev A (2017) Recalcitrant aseptic atrophic non-union of the shaft of the humerus after failure of surgical treatment: management by excision of non-union, bone grafting and stabilization by LCP in different modes. Injury 48 Suppl 2: S33-S43.

Baraniak PR, McDevitt TC (2010) Stem cell paracrine actions and tissue regeneration. Regen Med 5: 121-143.

Betsch M, Schneppendahl J, Thuns S, Herten M, Sager M, Jungbluth P, Hakimi M, Wild M (2013) Bone marrow aspiration concentrate and platelet rich plasma for osteochondral repair in a porcine osteochondral defect model. PloS One 8: e71602. DOI: 10.1371/journal.pone.0071602.

Boxall S, Jones E (2015) The use of multiparameter flow cytometry and cell sorting to characterize native human bone marrow mesenchymal stem cells (MSC). Methods Mol Biol 1235: 121-130.

Boxall SA, Jones E (2012) Markers for characterization of bone marrow multipotential stromal cells. Stem cells international 2012: 975871. DOI: 10.1155/2012/975871.

Calori GM, Giannoudis PV (2011) Enhancement of fracture healing with the diamond concept: the role of the biological chamber. Injury 42: 1191-1193.

Capelli C, Pedrini O, Valgardsdottir R, Da Roit F, Golay J, Introna M (2015) Clinical grade expansion of MSCs. Immunol Lett 168: 222-227.

Caplan AI, Dennis JE (2006) Mesenchymal stem cells as trophic mediators. J Cell Biochem 98: 10761084.

Cassano JM, Kennedy JG, Ross KA, Fraser EJ, Goodale MB, Fortier LA (2018) Bone marrow concentrate and platelet-rich plasma differ in cell distribution and interleukin 1 receptor antagonist protein concentration. Knee Surg Sports Traumatol Arthrosc 26: 333-342.

Castro-Malaspina H, Gay RE, Resnick G, Kapoor N, Meyers P, Chiarieri D, McKenzie S, Broxmeyer HE, Moore MA (1980) Characterization of human bone marrow fibroblast colony-forming cells (CFU-F) and their progeny. Blood 56: 289-301.

Chang J, Park H, Chae H, Kim M, Kim Y, Han $\mathrm{K}$ (2010) [Bone marrow cellularity measurement by myelocrit]. Korean J Lab Med 30: 224-230.

Churchman SM, Ponchel F, Boxall SA, Cuthbert R, Kouroupis D, Roshdy T, Giannoudis PV, Emery P, McGonagle D, Jones EA (2012) Transcriptional profile of native CD271+ multipotential stromal cells: evidence for multiple fates, with prominent osteogenic and Wnt pathway signaling activity. Arthritis Rheum 64: 2632-2643.

Connolly J, Guse R, Lippiello L, Dehne R (1989) Development of an osteogenic bone-marrow preparation. J Bone Joint Surg Am 71: 684-691.

Coppens DGM, De Bruin ML, Leufkens HGM, Hoekman J (2018) global regulatory differences for gene- and cell-based therapies: consequences and implications for patient access and therapeutic innovation. Clin Pharmacol Ther 103: 120-127.

Cuthbert R, Boxall SA, Tan HB, Giannoudis PV, McGonagle D, Jones E (2012) Single-platform quality control assay to quantify multipotential stromal cells in bone marrow aspirates prior to bulk manufacture or direct therapeutic use. Cytotherapy 14: 431-440.

Cuthbert RJ, Giannoudis PV, Wang XN, Nicholson L, Pawson D, Lubenko A, Tan HB, Dickinson A, McGonagle D, Jones E (2015) Examining the feasibility of clinical grade CD271+ enrichment of mesenchymal stromal cells for bone regeneration. PloS One 10: e0117855. DOI: 10.1371/journal.pone.0117855.

Dominici M, Le Blanc K, Mueller I, SlaperCortenbach I, Marini F, Krause D, Deans R, Keating A, Prockop D, Horwitz E (2006) Minimal criteria for defining multipotent mesenchymal stromal cells. The International Society for Cellular Therapy position statement. Cytotherapy 8: 315-317. 
Douras P, Tosounidis T, Giannoudis PV (2018) Application of the 'diamond concept' with fast bone marrow aspirate concentration for the treatment of medial malleolus non-union. Injury 49: 2326-2330.

El-Jawhari JJ, Jones E, Giannoudis PV (2016) The roles of immune cells in bone healing; what we know, do not know and future perspectives. Injury 47: 23992406.

El-Jawhari JJ, Cuthbert R, McGonagle D, Jones E, Giannoudis PV (2017) The CD45 $5^{\text {low }}$ CD271 ${ }^{\text {high }}$ cell prevalence in bone marrow samples may provide a useful measurement of the bone marrow quality for cartilage and bone regenerative therapy. J Bone Joint Surg Am 99: 1305-1313.

El-Jawhari JJ, Kleftouris G, El-Sherbiny Y, Saleeb H, West RM, Jones E, Giannoudis PV (2019a) Defective proliferation and osteogenic potential with altered immunoregulatory phenotype of native bone marrow-multipotential stromal cells in atrophic fracture non-union. Sci Rep 9: 17340. DOI: 10.1038/ s41598-019-53927-3.

El-Jawhari JJ, Moisley K, Jones E, Giannoudis PV (2019b) A crosslinked collagen membrane versus a non-crosslinked bilayer collagen membrane for supporting osteogenic functions of human bone marrow-multipotent stromal cells. Eur Cell Mater 37: 292-309.

Elahi KC, Klein G, Avci-Adali M, Sievert KD, MacNeil S, Aicher WK (2016) Human mesenchymal stromal cells from different sources diverge in their expression of cell surface proteins and display distinct differentiation patterns. Stem cells international 2016: 5646384. DOI: 10.1155/2016/5646384.

Ferrara N, Gerber HP (2001) The role of vascular endothelial growth factor in angiogenesis. Acta Haematol 106: 148-156.

Fragkakis EM, El-Jawhari JJ, Dunsmuir RA, Millner PA, Rao AS, Henshaw KT, Pountos I, Jones E, Giannoudis PV (2018) Vertebral body versus iliac crest bone marrow as a source of multipotential stromal cells: comparison of processing techniques, tri-lineage differentiation and application on a scaffold for spine fusion. PloS One 13: e0197969. DOI: 10.1371/journal. pone.0197969.

Friedenstein AJ (1980) Stromal mechanisms of bone marrow: cloning in vitro and retransplantation in vivo. Haematol Blood Transfus 25: 19-29.

Gao F, Chiu SM, Motan DA, Zhang Z, Chen L, Ji HL, Tse HF, Fu QL, Lian Q (2016) Mesenchymal stem cells and immunomodulation: current status and future prospects. Cell Death Dis 7: e2062. DOI: 10.1038/cddis.2015.327.

Gaul F, Bugbee WD, Hoenecke HR Jr, D'Lima DD (2019) A review of commercially available pointof-care devices to concentrate bone marrow for the treatment of osteoarthritis and focal cartilage lesions. Cartilage 10: 387-394.

Gessmann J, Koller M, Godry H, Schildhauer TA, Seybold D (2012) Regenerate augmentation with bone marrow concentrate after traumatic bone loss. Orthop Rev (Pavia) 4: e14. DOI: 10.4081/or.2012.e14.
Giannoudis PV, Einhorn TA, Marsh D (2007) Fracture healing: the diamond concept. Injury 38 Suppl 4: S3-6.

Giannoudis PV, Ahmad MA, Mineo GV, Tosounidis TI, Calori GM, Kanakaris NK (2013) Subtrochanteric fracture non-unions with implant failure managed with the "Diamond" concept. Injury 44 Suppl 1: S76-81.

Giannoudis PV, GudipatiS, Harwood P, Kanakaris NK (2015) Long bone non-unions treated with the diamond concept: a case series of 64 patients. Injury 46 Suppl 8: S48-54.

Gobbi A, Karnatzikos G, Scotti C, Mahajan V, Mazzucco L, Grigolo B (2011) One-step cartilage repair with bone marrow aspirate concentrated cells and collagen matrix in full-thickness knee cartilage lesions: results at 2-year follow-up. Cartilage 2: 286299.

Gobbi A, Chaurasia S, Karnatzikos G, Nakamura N (2015) Matrix-induced autologous chondrocyte implantation versus multipotent stem cells for the treatment of large patellofemoral chondral lesions: a nonrandomized prospective trial. Cartilage 6: 82-97.

Hakimi M, Grassmann JP, Betsch M, Schneppendahl J, Gehrmann S, Hakimi AR, Kropil P, Sager M, Herten M, Wild M, Windolf J, Jungbluth P (2014) The composite of bone marrow concentrate and PRP as an alternative to autologous bone grafting. PloS One 9: e100143. DOI: 10.1371/journal. pone.0100143.

Hernigou P, Poignard A, Beaujean F, Rouard H (2005a) Percutaneous autologous bone-marrow grafting for nonunions. Influence of the number and concentration of progenitor cells. J Bone Joint Surg Am 87: 1430-1437.

Hernigou P, Poignard A, Manicom O, Mathieu G, Rouard H (2005b) The use of percutaneous autologous bone marrow transplantation in nonunion and avascular necrosis of bone. J Bone Joint Surg Br 87: 896-902.

Hernigou P, Homma Y, Flouzat Lachaniette $\mathrm{CH}$, Poignard A, Allain J, Chevallier N, Rouard H (2013) Benefits of small volume and small syringe for bone marrow aspirations of mesenchymal stem cells. Int Orthop 37: 2279-2287.

Hoff P, Gaber T, Schmidt-Bleek K, Senturk U, Tran CL, Blankenstein K, Lutkecosmann S, Bredahl J, Schuler HJ, Simon P, Wassilew G, Unterhauser F, Burmester GR, Schmidmaier G, Perka C, Duda GN, Buttgereit $F$ (2011) Immunologically restricted patients exhibit a pronounced inflammation and inadequate response to hypoxia in fracture hematomas. Immunol Res 51: 116-122.

Holmes K, Roberts OL, Thomas AM, Cross MJ (2007) Vascular endothelial growth factor receptor-2: structure, function, intracellular signalling and therapeutic inhibition. Cell Signal 19: 2003-2012.

Holton J, Imam M, Ward J, Snow M (2016) The basic science of bone marrow aspirate concentrate in chondral injuries. Orthop Rev (Pavia) 8: 6659. DOI: 10.4081/or.2016.6659. 
Imam MA, Holton J, Ernstbrunner L, Pepke W, Grubhofer F, Narvani A, Snow M (2017) A systematic review of the clinical applications and complications of bone marrow aspirate concentrate in management of bone defects and nonunions. International orthopaedics 41: 2213-2220.

Jager M, Herten M, Fochtmann U, Fischer J, Hernigou P, Zilkens C, Hendrich C, Krauspe R (2011) Bridging the gap: bone marrow aspiration concentrate reduces autologous bone grafting in osseous defects. J Orthop Res 29: 173-180.

Jones E, McGonagle D (2008) Human bone marrow mesenchymal stem cells in vivo. Rheumatology (Oxford) 47: 126-131.

Jones E, Schafer R (2015) Where is the common ground between bone marrow mesenchymal stem/ stromal cells from different donors and species? Stem Cell Res Ther 6: 143. DOI: 10.1186/s13287-015-0144-8.

Kanakeshwar RB, Jayaramaraju D, Agraharam D, Rajasekaran S (2017) Management of resistant distal femur non-unions with allograft strut and autografts combined with osteosynthesis in a series of 22 patients. Injury 48 Suppl 2: S14-S17.

Kovach TK, Dighe AS, Lobo PI, Cui Q (2015) Interactions between MSCs and immune cells: implications for bone healing. J Immunol Res 2015: 752510. DOI: 10.1155/2015/752510.

Kucera J, Netusilova J, Sladecek S, Lanova M, Vasicek O, Stefkova K, Navratilova J, Kubala L, Pachernik J (2017) Hypoxia downregulates MAPK/ ERK but not STAT3 signaling in ROS-dependent and HIF-1-independent manners in mouse embryonic stem cells. Oxid Med Cell Longev 2017: 4386947. DOI: 10.1155/2017/4386947.

Lee DH, Ryu KJ, Kim JW, Kang KC, Choi YR (2014) Bone marrow aspirate concentrate and plateletrich plasma enhanced bone healing in distraction osteogenesis of the tibia. Clin Orthop Relat Res 472: 3789-3797.

Lienau J, Schmidt-Bleek K, Peters A, Weber H, Bail HJ, Duda GN, Perka C, Schell H (2010) Insight into the molecular pathophysiology of delayed bone healing in a sheep model. Tissue engineering. Part A 16: 191-199.

Moatshe G, Morris ER, Cinque ME, PascualGarrido C, Chahla J, Engebretsen L, Laprade RF (2017) Biological treatment of the knee with plateletrich plasma or bone marrow aspirate concentrates. Acta Orthop 88: 670-674.

Muschler GF, Nitto H, Boehm CA, Easley KA (2001) Age- and gender-related changes in the cellularity of human bone marrow and the prevalence of osteoblastic progenitors. J Orthop Res 19: 117-125.

Pak J, Lee JH, Park KS, Jeon JH, Lee SH (2017) Potential use of mesenchymal stem cells in human meniscal repair: current insights. Open Access J Sports Med 8: 33-38.

Piuzzi NS, Chahla J, Schrock JB, LaPrade RF, Pascual-Garrido C, Mont MA, Muschler GF (2017)
Evidence for the use of cell-based therapy for the treatment of osteonecrosis of the femoral head: a systematic review of the literature. J Arthroplasty 32: 1698-1708.

Schafer R, DeBaun MR, Fleck E, Centeno CJ, Kraft D, Leibacher J, Bieback K, Seifried E, Dragoo JL (2019) Quantitation of progenitor cell populations and growth factors after bone marrow aspirate concentration. J Transl Med 17: 115. DOI: 10.1186/ s12967-019-1866-7.

Schieber M, Chandel NS (2014) ROS function in redox signaling and oxidative stress. Curr Biol 24: R453-462.

Siegel G, Kluba T, Hermanutz-Klein U, Bieback K, Northoff H, Schafer R (2013) Phenotype, donor age and gender affect function of human bone marrowderived mesenchymal stromal cells. BMC Med 11: 146. DOI: 10.1186/1741-7015-11-146

Smyth NA, Murawski CD, Haleem AM, Hannon CP, Savage-Elliott I, Kennedy JG (2012) Establishing proof of concept: platelet-rich plasma and bone marrow aspirate concentrate may improve cartilage repair following surgical treatment for osteochondral lesions of the talus. World J Orthop 3: 101-108.

Song L, Tuan RS (2004) Transdifferentiation potential of human mesenchymal stem cells derived from bone marrow. FASEB J 18: 980-982.

Takeshima T, Yumura Y, Kuroda S, Kawahara T, Uemura H, Iwasaki A (2017) Effect of density gradient centrifugation on reactive oxygen species in human semen. Syst Biol Reprod Med 63: 192-198.

Tomlinson MJ, Tomlinson S, Yang XB, Kirkham J (2013) Cell separation: terminology and practical considerations. J Tissue Eng 4: 2041731412472690. DOI: $10.1177 / 2041731412472690$.

Tormin A, Li O, Brune JC, Walsh S, Schutz B, Ehinger M, Ditzel N, Kassem M, Scheding S (2011) CD146 expression on primary nonhematopoietic bone marrow stem cells is correlated with in situ localization. Blood 117: 5067-5077.

Verboket R, Leiblein M, Seebach C, Nau C, Janko M, Bellen M, Bonig H, Henrich D, Marzi I (2018) Autologous cell-based therapy for treatment of large bone defects: from bench to bedside. Eur J Trauma Emerg Surg 44: 649-665.

Woodell-May JE, Tan ML, King WJ, Swift MJ, Welch ZR, Murphy MP, McKale JM (2015) Characterization of the cellular output of a pointof-care device and the implications for addressing critical limb ischemia. Biores Open Access 4: 417-424.

Zellner J, Taeger CD, Schaffer M, Roldan JC, Loibl M, Mueller MB, Berner A, Krutsch W, Huber MK, Kujat R, Nerlich M, Angele P (2014) Are applied growth factors able to mimic the positive effects of mesenchymal stem cells on the regeneration of meniscus in the avascular zone? Biomed Res Int 2014: 537686. DOI: $10.1155 / 2014 / 537686$.

Zhong W, Sumita Y, Ohba S, Kawasaki T, Nagai K, Ma G, Asahina I (2012) In vivo comparison of the bone regeneration capability of human bone marrow 
concentrates vs. platelet-rich plasma. PloS One 7: e40833. DOI: 10.1371/journal.pone.0040833.

Zwaginga JJ, Doevendans P (2003) Stem cellderived angiogenic/vasculogenic cells: possible therapies for tissue repair and tissue engineering. Clin Exp Pharmacol Physiol 30: 900-908.

\section{Discussion with Reviewer}

Reviewer: The manuscript reported on a vertical centrifugation system resulting in 4-fold enrichment in multipotent stromal cells. At the same time, data also suggested enrichment of platelets and leukocytes. Could the authors speculate which influence this might have on the regenerative potential of the BM concentrate when directly applied to a defect site in an intra-operative setting?

Authors: The enriched MSCs and platelets in BM concentrate would be of great potential value for the healing of the bone/cartilage defect. The enriched MSCs would have an increased osteogenic and chondrogenic effect. Additionally, enriched platelets release more of the growth factors supporting the proliferation and survival of MSCs and enhancing their reparatory impact. The leucocytes present in PRP preparations did not seem to change the proliferation or migration of MSCs, as reported before (Moisley et al., 2019, additional reference). However, the outcome of the enriched leukocytes in $\mathrm{BM}$ concentrate on the healing process would require further investigations. This cell cocktail of BM concentrate has the advantages of being quickly processed within theatre time and being autologous, with no expected immunogenic adverse effects.

\section{Additional Reference}

Moisley KM, El-Jawhari JJ, Owston H, Tronci G, Russell SJ, Jones EA, Giannoudis PV (2019)Optimising proliferation and migration of mesenchymal stem cells using platelet products: A rational approach to bone regeneration. J Orthop Res 37: 1329-1338.

Editor's note: The Scientific Editor responsible for this paper was Martin Stoddart. 